\title{
A cooperative tumour cell community
}

The concept of intratumour heterogeneity is now well established. However, it is unclear whether tumour cell subclones compete or cooperate with each other during the course of tumour progression and whether heterogeneity in tumour cell populations is a cause, rather than a consequence, of tumour development. Using mouse models of breast cancer, Edward Gunther and colleagues have found that functional cooperation occurs between unique subclones within an individual tumour.

Mammary tumours in mice expressing WNT1 from the mouse mammary tumour virus (MMTV) promoter are thought to originate from a bipotent mammary progenitor cell that produces basal and luminal cells, and half of these tumours spontaneously acquire somatic Hras mutations (Hras $\left.{ }^{m u t}\right)$. However, contrary to an expected mutant allele fraction (MAF) of 0.5 for dominant Hras $^{\text {mut }}$, the authors observed a MAF of $<0.3$ in several tumours. This low MAF was maintained if tumours were explanted into syngeneic host mice and was not a result of contamination with non-tumour cells. Tumour cell sorting indicated
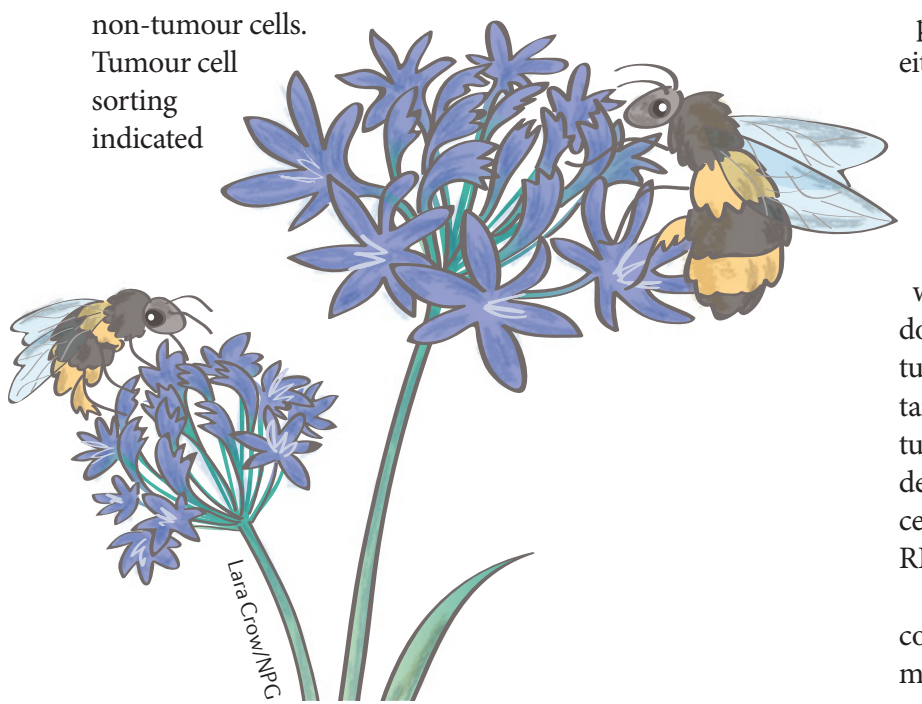

that five of ten MMTV-WNT1 Hras ${ }^{\text {mut }}$ tumours had an Hras $^{\text {mut }}$ MAF $>0.5$ and both basal and luminal cells carried Hras $^{\text {mut }}$, suggesting a hierarchical configuration wherein tumour cells derived from a common progenitor. The other five tumours had an $\mathrm{Hras}^{\text {mut }}$ MAF $<0.5$, and Hras $^{\text {mut }}$ was enriched only in the basal tumour cells, which is consistent with biclonality. Furthermore, these tumours had mixed-lineage histopathology, with only luminal tumour cells expressing WNT1, indicating that these tumours comprised two subclones: that is, Hras $^{\text {mut }}$ Wnt ${ }^{\text {low }}$ and Hras ${ }^{\text {wt }}$ Wnt $1^{\text {high }}$.

To experimentally address whether cooperation between subclones is required in some tumours, the authors used biclonal tumour cells from mice expressing red fluorescent protein (RFP) and a doxycycline-inducible WNT1 (iWNT1) to recon-

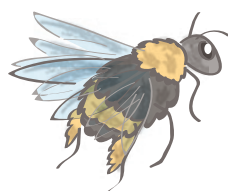
stitute tumours in host mice that either did not express WNT1 (control hosts) or that expressed WNT1 constitutively (cWNT1 hosts). Orthotopic injection of the iWNT1 RFPpositive $\left(\mathrm{RFP}^{+}\right)$tumour cells into either strain of host mouse resulted in tumours in the presence of doxycycline. When iWNT1 was turned off by doxycycline withdrawal in control hosts, tumours regressed but could relapse if doxycycline was reintroduced. In cWNT1 hosts, doxycycline withdrawal caused partial tumour regression and eventual spontaneous relapse, and these relapsed tumours comprised both donorderived Hras $^{\text {mut }}$ Wnt $1^{\text {low }} \mathrm{RFP}^{+}$basal cells and host-derived Hras ${ }^{w t}$ Wnt $1^{\text {high }}$ RFP-negative luminal tumour cells.

Having established that clonal cooperation can occur in this mouse model, the authors then further examined spontaneously relapsed, doxycyclineindependent iWNT1 tumours as a model of acquired resistance following therapy. Analysis of 20 doxycycline-independent relapsed tumours indicated that most had reactivated the WNT pathway in one of two ways. The first set acquired mutations in the reverse tetracycline-controlled transactivator (rtTA) that allowed doxycyclineindependent expression of the Wnt 1 transgene; these tumours maintained biclonality, suggesting that the $r t T A$ mutations arose in the $\mathrm{Hras}^{\mathrm{wt}}$ cells. The second set relapsed through acquiring mutations in the gene encoding $\beta$-catenin (Ctnnb1), which functions downstream of WNT1. Interestingly, these relapsed tumours had an increased $\mathrm{Hras}^{\text {mut }}$ MAF, which suggests that the Ctnnb1 mutation arose in the Hras ${ }^{m u t}$ cells and that these tumours no longer require cooperation between subclones.

Therefore, tumours might maintain heterogeneity through either a differentiation hierarchy or because of the necessity for cooperation between lineage-restricted subclones. Hierarchical and biclonal tumours in the MMTV-WNT1 model are histologically similar, but it remains to be determined whether they might have different clinical behaviour. As WNT1 is a secreted factor, it will also be interesting to determine whether subclonal cooperation can arise in cases where an oncogene functions cell-intrinsically.

Sarah Seton-Rogers

ORIGINAL RESEARCH PAPER Cleary, A. S. et al. Tumour cell heterogeneity maintained by cooperating subclones in Wnt-driven mammary cancers. Nature 508, 113-117 (2014) 